\title{
NODAL GEOMETRY ON RIEMANNIAN MANIFOLDS
}

\author{
SAGUN CHANILLO \& B. MUCKENHOUPT
}

1. Let $M^{n}$ be a smooth, compact, and connected Riemannian manifold with no boundary. Let $\Delta$ denote the Laplacian on $M$. Let $-\Delta u=$ $\lambda u, u$ an eigenfunction with eigenvalue $\lambda, \lambda>1$. Our main theorems are:

Theorem 1 (BMO estimate for $\log |u|)$. For $u, \lambda$ as above,

$$
\|\log |u|\|_{\mathrm{BMO}} \leq c \lambda^{n} \log \lambda,
$$

where $c$ is independent of $\lambda$, and depends only on $n$ and $M$.

Theorem 2 (Geometry of nodal domains). Let $u, \lambda$ be as above, let $B \subset M$ be any ball, and let $\Omega \subset B$ be any of the connected components of $\{x \in B: u(x) \neq 0\}$. If $\Omega$ intersects the middle half of $B$, then

$$
|\Omega| \geq c \lambda^{-2 n^{2}-n / 2}(\log \lambda)^{-2 n}|B|,
$$

where $c$ is independent of $\lambda$ and $u$.

Similar theorems have been proved by $\mathrm{H}$. Donnelly and $\mathrm{C}$. Fefferman [1], [2] with $\lambda^{n} \log \lambda$ replaced by $\lambda^{n(n+2) / 4}$ in Theorem 1 and $\lambda^{-2 n^{2}-n / 2}(\log \lambda)^{-2 n}$ replaced by $\lambda^{-\left(n+n^{2}(n+2)\right) / 2}$ in Theorem 2. Of course, it is obvious that Theorems 1 and 2 above are not best possible.

Theorem 1 is the key to Theorem 2 . We deduce Theorem 2 from Theorem 1 by essentially following the arguments in [2] with appropriate modifications in view of the better BMO estimate of Theorem 1.

We shall use the symbols $c, c_{0}, c_{1}, c_{2}, c_{3}, c_{4}$, and $\bar{c}$ to denote generic constants which are independent of $\lambda$.

2. Before commencing the proof of Theorem 1, we recall two facts from [2]. We state these as Theorem 0 .

Theorem 0. Let $M, u, \lambda$ be as above. Let $B(x, \delta)$ denote the ball centered at $x$ of radius $\delta$. Then

Received December 14, 1989. The first author was supported in part by National Science Foundation grant DMS88-03493. 
(A)

$$
\begin{gathered}
\int_{B\left(x, \delta\left(1+\lambda^{-1 / 2}\right)\right)}|u|^{2} \leq c \int_{B(x, \delta)}|u|^{2}, \\
\left(\int_{B(x, \delta)}|\nabla u|^{2}\right)^{1 / 2} \leq c \frac{\sqrt{\lambda}}{\delta}\left(\int_{B(x, \delta)}|u|^{2}\right)^{1 / 2} .
\end{gathered}
$$

We now begin the proof of Theorem 1 .

Lemma 1. Let $u, \lambda$ be as before. Then $u$ satisfies the reverse-Holder inequality,

$$
\left(\frac{1}{|B|} \int_{B}|u|^{2 n /(n-2)}\right)^{(n-2) / 2 n} \leq c \sqrt{\lambda}\left(\frac{1}{|B|} \int_{B}|u|^{2}\right)^{1 / 2} .
$$

Proof. By the Poincaré-Sobolev inequality, for any ball $B$,

$$
\left(\frac{1}{|B|} \int_{B}\left|u-\frac{1}{|B|} \int_{B} u\right|^{2 n /(n-2)}\right)^{(n-2) / 2 n} \leq c|B|^{1 / n}\left(\frac{1}{|B|} \int_{B}|\nabla u|^{2}\right)^{1 / 2} .
$$

We now apply Theorem $0(B)$ to the right side above, to get

$$
\left(\frac{1}{|B|} \int_{B}\left|u-\frac{1}{|B|} \int_{B} u\right|^{2 n /(n-2)}\right)^{(n-2) / 2 n} \leq c \sqrt{\lambda}\left(\frac{1}{|B|} \int_{B}|u|^{2}\right)^{1 / 2} \text {. }
$$

By use of Minkowski's inequality, Lemma 1 follows. q.e.d.

Our theorem will follow from the lemma stated below.

Lemma 2. Suppose $w>0$,

$$
\int_{B(x, \delta(1+1 / \sqrt{\lambda}))} w \leq c_{0} \int_{B(x, \delta)} w
$$

and

$$
\left(\frac{1}{|B|} \int_{B} w^{n /(n-2)}\right)^{(n-2) / n} \leq c_{1} \lambda \frac{1}{|B|} \int_{B} w
$$

Then $\|\log w\|_{\mathrm{BMO}} \leq c(n) \lambda^{n} \log \lambda$.

Theorem 1 follows by choosing $w=|u|^{2}$.

Our next lemma is a covering lemma of independent interest.

Lemma 3. Fix any $\delta>0$, with $\delta<1 / 2$. Let $\left\{B_{\alpha}\right\}_{\alpha \in I}$ be any finite collection of balls in $\mathbb{R}^{n}$. Then one can find a subcollection of balls $B_{1}, B_{2}, \cdots, B_{N}$ such that

$$
\begin{gathered}
\bigcup_{\alpha \in I} B_{\alpha} \subset \bigcup_{i=1}^{N}(1+\delta) B_{i}, \\
\sum_{i=1}^{N} \chi_{B_{i}}(x) \leq 4^{n} \delta^{-n} .
\end{gathered}
$$


Proof. Select a ball $B_{1}$ with the largest radius from the collection $\left\{B_{\alpha}\right\}_{\alpha \in I}$. Having selected $B_{1}, \cdots, B_{k-1}$ select $B_{k}$ so that $B_{k} \not \subset$ $\bigcup_{i=1}^{k-1}(1+\delta) B_{i}$ and $B_{k}$ has the largest possible radius out of the balls in the collection $\left\{B_{\alpha}\right\}_{\alpha \in I} \backslash\left\{B_{i}\right\}_{i=1}^{k-1}$. Our choice of the subcollection $B_{1}, \cdots, B_{N}$ clearly satisfies (a). We now prove (b). Let $x_{0} \in \bigcap_{i=1}^{M} B_{i}, M=M\left(x_{0}\right)$. By a translation we may suppose $x_{0}=0$. For $x \in \mathbb{R}^{n}$, define $T_{r}(x)=x / r$, $r>0$. By our selection procedure $T_{r_{k}}\left(B_{k}\right) \not \subset \bigcup_{i=1}^{k-1}(1+\delta) T_{r_{k}}\left(B_{i}\right)$, where $r_{i}$ denotes the radius of $B_{i}$. Now $T_{r_{k}}\left(B_{i}\right)$ is also a ball containing the origin, and since $r_{i} \geq r_{k}$ for $i<k$, we have $T_{r_{i}}\left(B_{i}\right) \subset T_{r_{k}}\left(B_{i}\right)$, and we conclude

$$
T_{r_{k}}\left(B_{k}\right) \not \subset \bigcup_{i=1}^{k-1}(1+\delta) T_{r_{i}}\left(B_{i}\right) .
$$

Let $z_{i}$ denote the center of $T_{r_{i}}\left(B_{i}\right)$. We note that each of the balls $T_{r_{i}}\left(B_{i}\right)$ has radius 1 and $0 \in \bigcap_{i=1}^{M} T_{r_{i}}\left(B_{i}\right)$. We will show that $\left|z_{i}-z_{j}\right| \geq \delta$. For if $\left|z_{i}-z_{j}\right|<\delta$, and assuming $r_{i} \geq r_{j}$, we get $(1+\delta) T_{r_{i}}\left(B_{i}\right) \supset T_{r_{j}}\left(B_{j}\right)$, a violation of $(*)$. Thus the balls $B\left(z_{i}, \delta / 2\right)$ are all disjoint. Furthermore $T_{r_{i}}\left(B_{i}\right) \subset\{x:|x|<2\}$ for all $i=1,2, \cdots, M$. Hence, $M(\delta / 2)^{n} \leq 2^{n}$, i.e., $M \leq 4^{n} \delta^{-n}$, and (b) follows.

Lemma 4. Let $w$ satisfy the hypothesis of Lemma 2, let $B$ be a fixed ball, and let $E \subset B$ such that $|E| \geq\left(1-c_{2} \lambda^{-n}\right)^{k}|B|$. Then

$$
\int_{E} w \geq\left(c_{3} \lambda^{-n / 2}\right)^{k} \int_{B} w
$$

where $c_{2}=c_{2}\left(n, c_{1}\right)$ and $c_{3}=c_{3}\left(n, c_{0}\right)$.

Proof. The proof of Lemma 4 rests on an induction on $k$, the inductive step being accomplished by Lemma 3 . We verify Lemma 4 for $k=1$. To do so note that if $|E| \geq\left(1-\bar{c} \lambda^{-n / 2}\right)|B|$ (for some appropriate choice of $\left.\bar{c}=\bar{c}\left(c_{1}, n\right)\right)$, then $\int_{E} w \geq \frac{1}{2} \int_{B} w$. To see this, observe $|B \backslash E| \leq$ $\bar{c} \lambda^{-n / 2}|B|$. Thus by (2.2),

$$
\int_{B \backslash E} w \leq\left(\int_{B} w^{n /(n-2)}\right)^{(n-2) / n}|B \backslash E|^{2 / n} \leq \bar{c}^{2 / n} c_{1} \int_{B} w .
$$

We make the choice $\bar{c}^{2 / n} c_{1}<1 / 2$ and inserting this choice into the inequality above we get $\int_{B \backslash E} w \leq \frac{1}{2} \int_{B} w$. Thus $\int_{E} w \geq \frac{1}{2} \int_{B} w$. If $c_{2} \leq \bar{c}$ and $|E| \geq\left(1-c_{2} \lambda^{-n}\right)|B|$, then $|E| \geq\left(1-\bar{c} \lambda^{-n / 2}\right)|B|$. Therefore $\int_{E} w \geq \frac{1}{2} \int_{B} w \geq c_{3} \lambda^{-n / 2} \int_{B} w$, and we are done with the case $k=1$. 
So we assume the statements are valid for $k-1$. Clearly we can assume $|E| \leq\left(1-\bar{c} \lambda^{-n / 2}\right)|B|$ or else there is nothing to prove. For each point of density $x$ of $E$ we can thus select a ball $B_{x} \subset B$ such that $x \in B_{x}$ and

$$
\left|B_{x} \cap E\right| /\left|B_{x}\right|=1-\bar{c} \lambda^{-n / 2} .
$$

We apply the covering lemma, Lemma 3 , to the $B_{x}$ 's with the choice $\delta=\lambda^{-1 / 2}$, and also assume without loss of generality that the $B_{x}$ are finitely many. Define $E_{1}=\left(\bigcup_{i=1}^{N}\left(1+\lambda^{-1 / 2}\right) B_{i}\right) \cap B$. Then $E_{1} \subset B$, and to complete the induction we will show that

$$
\begin{gathered}
|E| \leq\left(1-c_{2} \lambda^{-n}\right)\left|E_{1}\right|, \\
\int_{E} w \geq c_{3} \lambda^{-n / 2} \int_{E_{1}} w .
\end{gathered}
$$

We prove (2.3) first. Now,

$$
\begin{aligned}
\left|E_{1}\right| & =|E|+\left|\left(\bigcup\left(1+\lambda^{-1 / 2}\right) B_{i}\right) \cap B \backslash E\right| \\
& \geq|E|+\left|\left(\bigcup B_{i}\right) \cap B \backslash E\right|=|E|+\left|\bigcup B_{i} \backslash E\right| .
\end{aligned}
$$

By the covering lemma, Lemma 3, the expression above is bounded below by

$$
|E|+4^{-n} \lambda^{-n / 2} \sum\left|B_{i} \backslash E\right| .
$$

By our selection, $\left|B_{i} \backslash E\right|=\bar{c} \lambda^{-n / 2}\left|B_{i}\right|$, thus (2.5) is bounded below by

$$
|E|+\sum \bar{c} 4^{-n} \lambda^{-n}\left|B_{i}\right|=|E|+\bar{c} 4^{-n}\left(1+\lambda^{-1 / 2}\right)^{-n} \lambda^{-n} \sum\left|\left(1+\lambda^{-1 / 2}\right) B_{i}\right| .
$$

Set $\bar{c} 4^{-n}\left(1+\lambda^{-1 / 2}\right)^{-n}=c_{2}$, and note $c_{2} \leq \bar{c}$. From the expression above we deduce

and (2.3) follows.

$$
\left|E_{1}\right| \geq c_{2} \lambda^{-n}\left|E_{1}\right|+|E|
$$

We now prove (2.4). By (2.1),

$$
\int_{E_{1}} w \leq \sum \int_{\left(1+\lambda^{-1 / 2}\right) B_{i}} w \leq c_{0} \sum \int_{B_{i}} w
$$

But $\left(1-\bar{c} \lambda^{-n / 2}\right)\left|B_{i}\right|=\left|E \cap B_{i}\right|$, thus $\int_{B_{i}} w \leq 2 \int_{B_{i} \cap E} w$. Therefore, by Lemma 3,

$$
c_{0} \sum \int_{B_{i}} w \leq 2 c_{0} \sum \int_{B_{i} \cap E} w \leq 2 c_{0} \int_{E} w \sum \chi_{B_{i}} \leq 2 \cdot 4^{n} c_{0} \lambda^{n / 2} \int_{E} w .
$$

We select $c_{3}^{-1}=2 \cdot 4^{n} c_{0}$ and (2.4) follows. q.e.d. 
We now prove Lemma 2 . It will be enough to assume $|B|^{-1} \int_{B} w=1$, and to show for $t>0$

$$
\left|\left\{x \in B: w^{-1}(x)>t\right\}\right| \leq \frac{|B|}{t^{c \lambda^{-n}(\log \lambda)^{-1}}},
$$

which is equivalent to showing

$$
|\{x \in B: w(x)<t\}| \leq t^{c \lambda^{-n}(\log \lambda)^{-1}}|B| .
$$

Let us denote by $E$ the set $\{x \in B: w(x)<t\}$. Select $k_{0}$ such that $|E| \sim\left(1-c_{2} \lambda^{-n}\right)^{k_{0}}|B|$. Thus $k_{0} \sim c \lambda^{n} \log (|B| /|E|)$, and so by Lemma 4 and the normalization $|B|^{-1} \int_{B} w=1$, we get,

$$
|B|=\int_{B} w \leq\left(c_{3} \lambda^{n / 2}\right)^{k_{0}} \int_{E} w \leq\left(c_{3} \lambda^{n / 2}\right)^{k_{0}} t|E| \text {. }
$$

Hence,

$$
|B| /|E| \leq t e^{c k_{0} \log \lambda^{n / 2}} \leq t(|B| /|E|)^{c \lambda^{n} \log \lambda}
$$

and it follows easily that $|E| \leq t^{c \lambda^{-n}(\log \lambda)^{-1}}|B|$. q.e.d.

We now prove Theorem 2 . We will be brief and only indicate those points in our argument which differ substantially from the argument presented in [2]. Before commencing we note an equivalent formulation of Theorem 1:

Theorem $\mathbf{1}^{\prime}$. Let $u, \lambda$ be as before and let $E \subset B$. Then

$$
\sup _{B}|u| \leq(c|B| /|E|)^{c \lambda^{n} \log \lambda} \sup _{E}|u| \text {. }
$$

The lemma stated below is proved in [2] (Lemma 2 there).

Lemma 5. Suppose $\Omega$ is a component of $\left\{x \in B\left(x_{0}, \delta\right), u(x)>0\right\}$ and assume $x_{0} \in \Omega$ and $0<\delta<\lambda^{-1 / 2}$. Suppose further $|\Omega| /\left|B\left(x_{0}, \delta\right)\right|<$ $\eta^{n}<\frac{1}{2} \eta_{0}^{n}<\frac{1}{2}$. Then there is a positive number $r_{0}$ satisfying

$$
\begin{gathered}
0<r_{0}<\frac{\eta}{\eta_{0}} \delta, \\
\frac{\left|\Omega \cap B\left(x_{0}, r_{0}\right)\right|}{\left|B\left(x_{0}, r_{0}\right)\right|} \geq \eta_{0}^{n}, \\
\sup _{\Omega \cap B\left(x_{0}, r_{0}\right)}|u| \leq\left(\frac{r_{0}}{\delta}\right)^{c_{4} / \eta} \sup _{B\left(x_{0}, \delta\right)}|u|,
\end{gathered}
$$

where $c_{4}$ depends on the "bounded geometry" estimates. 
We also need the estimate below which is Theorem 1 in [1].

Lemma 6. Let $u, \lambda$ be as above. Then

$$
\sup _{B(x, r)}|u| \leq\left(c \frac{r}{r^{\prime}}\right)^{c \sqrt{\lambda}} \sup _{B\left(x, r^{\prime}\right)}|u|, \quad 0<r^{\prime}<r .
$$

Lemma 6 may be deduced from Theorem $0(A)$ above by an iteration and a use of the mean value inequalities for $u$.

Proof of Theorem 2. By Theorem 1',

$$
\begin{aligned}
\sup _{B\left(x_{0}, r_{0}\right)}|u| & \leq\left(\frac{c\left|B\left(x_{0}, r_{0}\right)\right|}{\left|\Omega \cap B\left(x_{0}, r_{0}\right)\right|}\right)^{c \lambda^{n} \log \lambda} \sup _{B\left(x_{0}, r_{0}\right) \cap \Omega}|u| \\
& \leq\left(c \eta_{0}^{-n}\right)^{c \lambda^{n} \log \lambda} \sup _{B\left(x_{0}, r_{0}\right) \cap \Omega}|u| .
\end{aligned}
$$

The estimate above follows by assuming $|\Omega| /\left|B\left(x_{0} ; \delta\right)\right| \leq \eta^{n}$ and then using Lemma $5(\mathrm{~b})$. We shall arrive at a contradiction for a suitable choice of $\eta$, and thus for this choice of $\eta$ we will have $|\Omega| /\left|B\left(x_{0}, \delta\right)\right| \geq \eta^{n}$ which will prove Theorem 2. Using Lemma 5(c) we get

$$
\left(c \eta_{0}^{-n}\right)^{c \lambda^{n} \log \lambda} \sup _{B\left(x_{0}, r_{0}\right) \cap \Omega}|u| \leq\left(c \eta_{0}^{-n}\right)^{c \lambda^{n} \log \lambda}\left(\frac{r_{0}}{\delta}\right)^{\delta / \eta} \sup _{B\left(x_{0}, \delta\right)}|u| .
$$

Thus,

$$
\sup _{B\left(x_{0}, r_{0}\right)}|u| \leq\left(c \eta_{0}^{-n}\right)^{c \lambda^{n} \log \lambda}\left(\frac{r_{0}}{\delta}\right)^{c_{4} / \eta} \sup _{B\left(x_{0}, \delta\right)}|u| .
$$

Applying Lemma 6 to the left side above yields

$$
\begin{aligned}
\sup _{B\left(x_{0}, \delta\right)}|u| & \leq\left(c \frac{\delta}{r_{0}}\right)^{c \sqrt{\lambda}}\left(c \eta_{0}^{-n}\right)^{c \lambda^{n} \log \lambda}\left(\frac{r_{0}}{\delta}\right)^{c_{4} / \eta} \sup _{B\left(x_{0}, \delta\right)}|u| \\
& \leq\left(c \frac{r_{0}}{\delta}\right)^{c_{4} / \eta-c \sqrt{\lambda}}\left(c \eta_{0}^{-n}\right)^{c \lambda^{n} \log \lambda} \sup _{B\left(x_{0}, \delta\right)}|u| .
\end{aligned}
$$

Therefore $\left(c r_{0} / \delta\right)^{c_{4} / \eta-c \sqrt{\lambda}}\left(c \eta_{0}^{-n}\right)^{c \lambda^{n} \log \lambda} \geq 1$.

Let us assume that our choice of $\eta$ is such that $c_{4} / \eta-c \sqrt{\lambda} \geq 0$. So using Lemma 5(a) we see easily

$$
1 \leq\left(c \frac{\eta}{\eta_{0}}\right)^{c_{4} / \eta-c \sqrt{\lambda}}\left(c \eta_{0}^{-n}\right)^{c \lambda^{n} \log \lambda} .
$$

We now choose $\eta=\eta_{0}^{2}$ and $\eta_{0}=\tilde{c} \lambda^{-n}(\log \lambda)^{-1}$. This choice forces $c_{4} / \eta-c \sqrt{\lambda} \geq 0$ and also yields

$$
1 \leq\left(c \eta_{0}\right)^{c_{4} / \eta-c \sqrt{\lambda}-c n \lambda^{n} \log \lambda} .
$$


This is a contradiction as $c \eta_{0}<1$ for small $\tilde{c}$. Thus, $|\Omega| /\left|B\left(x_{0}, \delta\right)\right|>$ $\eta^{n}=c \lambda^{-2 n}(\log \lambda)^{-2 n}$. We now get rid of the restriction $\delta<\lambda^{-1 / 2}$. Suppose $B \subset M$ is any ball with radius $r>\lambda^{-1 / 2}$. Assume $x_{0} \in \Omega$ belongs also to the middle half of $B$. We apply our previous conclusion to $\Omega \cap B\left(x_{0}, \lambda^{-1 / 2}\right)$ to get

$$
\begin{aligned}
|\Omega \cap B| & \geq\left|\Omega \cap B\left(x_{0}, \lambda^{-1 / 2}\right)\right| \geq c \lambda^{-2 n^{2}}(\log \lambda)^{-2 n}\left|B\left(x_{0}, \lambda^{-1 / 2}\right)\right| \\
& =\frac{c \lambda^{-2 n^{2}-n / 2}(\log \lambda)^{-2 n}}{r^{n}}|B| .
\end{aligned}
$$

But $r \leq c_{0}$ as the manifold is compact and we hence arrive at

$$
|\Omega \cap B| \geq c \lambda^{-2 n^{2}-n / 2}(\log \lambda)^{-2 n}|B| .
$$

\section{References}

[1] H. Donnelly \& C. Fefferman, Nodal sets of eigenfunctions on Riemannian manifolds, Invent. Math. 93 (1988) 161-183.

[2] __, Growth and geometry of eigenfunctions of the Laplacian, Analysis and Partial Differential Equations, Lecture Notes in Pure and Applied Math. vol. 122, M. Dekker, New York, 1990, 635-655. 
